\title{
Structures and Materials
}

Link to publication record in Manchester Research Explorer

\section{Citation for published version (APA):}

Tan, H., Hjelmstad, K., \& Geubelle, PH. (2006). Structures and Materials. In Center for Simulation of Advanced Rockets annual reports (2005- 2006) (in: Center for Simulation of Advanced Rockets annual reports (2005-2006): Structures and Materials). University of Illinois at Urbana-Champaign. http://www.csar.uiuc.edu/

\section{Published in:}

Center for Simulation of Advanced Rockets annual reports (2005- 2006)

\section{Citing this paper}

Please note that where the full-text provided on Manchester Research Explorer is the Author Accepted Manuscript or Proof version this may differ from the final Published version. If citing, it is advised that you check and use the publisher's definitive version.

\section{General rights}

Copyright and moral rights for the publications made accessible in the Research Explorer are retained by the authors and/or other copyright owners and it is a condition of accessing publications that users recognise and abide by the legal requirements associated with these rights.

\section{Takedown policy}

If you believe that this document breaches copyright please refer to the University of Manchester's Takedown Procedures [http://man.ac.uk/04Y6Bo] or contact uml.scholarlycommunications@manchester.ac.uk providing relevant details, so we can investigate your claim.

\section{OPEN ACCESS}




\subsection{Structures and Materials}

Group leaders: Keith Hjelmstad and Philippe Geubelle

Faculty: Robert Averback, Armand Beaudoin, Robert Dodds, C. Duarte, Philippe Geubelle, Keith Hjelmstad, Eric Loth, Yonggang Huang, Petros Sofronis, and Daniel Tortorelli

Research Scientists: Karel Matous, Alireza Namazifard, and Henry Tan

Research Programmers: M. Scott Breitenfeld

Post-doctoral Associates: Youhong Li

Graduate Research Assistants: K. Al-Fadhalah, Kristine Cochran (DOE Krell Fellow), Heath Dewey, Morgan Hawker, Mark Gates, Helen Inglis, Rajeev Jaiman, M. Kulkarni, N. Kumar, C. Liu, Justin Mach, S. Meyer, Kalyanbabu Nakshatrala, Praveen Nakshatrala, Henry Padilla, Arun Prakash, K. Srinivasan, Michael Tonks, Daniel Turner, Jay Patel, Brad Roe, Satya Varadhan, Fengbin Xu, Weixing Zhou

Undergraduate Research Assistants: Brett Collins, Jonathan Gu, Steven Stetak, and Jessica Kramer

\section{Overview}

The Structures and Materials group is responsible for the analysis of the solid parts of the rocket - the rocket case and the unburned solid fuel. The activities of this group divide into two thrust areas: (1) system simulation and (2) constitutive and failure modeling of components and materials. The technology developed in both of these general areas migrates, when appropriate, to the integrated simulation codes, primarily through the modules Rocsolid and Rocfrac. The research activities in system simulation primarily concern the groups led by Professors Dodds, Hjelmstad, and Tortorelli. The research activities in constitutive and failure modeling primarily concern the groups led by Professors Averback, Beaudoin, Geubelle, Huang, and Sofronis. In addition to these two key activities, the S\&M Team has been involved in integrated simulations of subscale multiphysics problems such as the convective burning of cracks in energetic materials and the mesoscale modeling of burning in a damaged solid propellant.
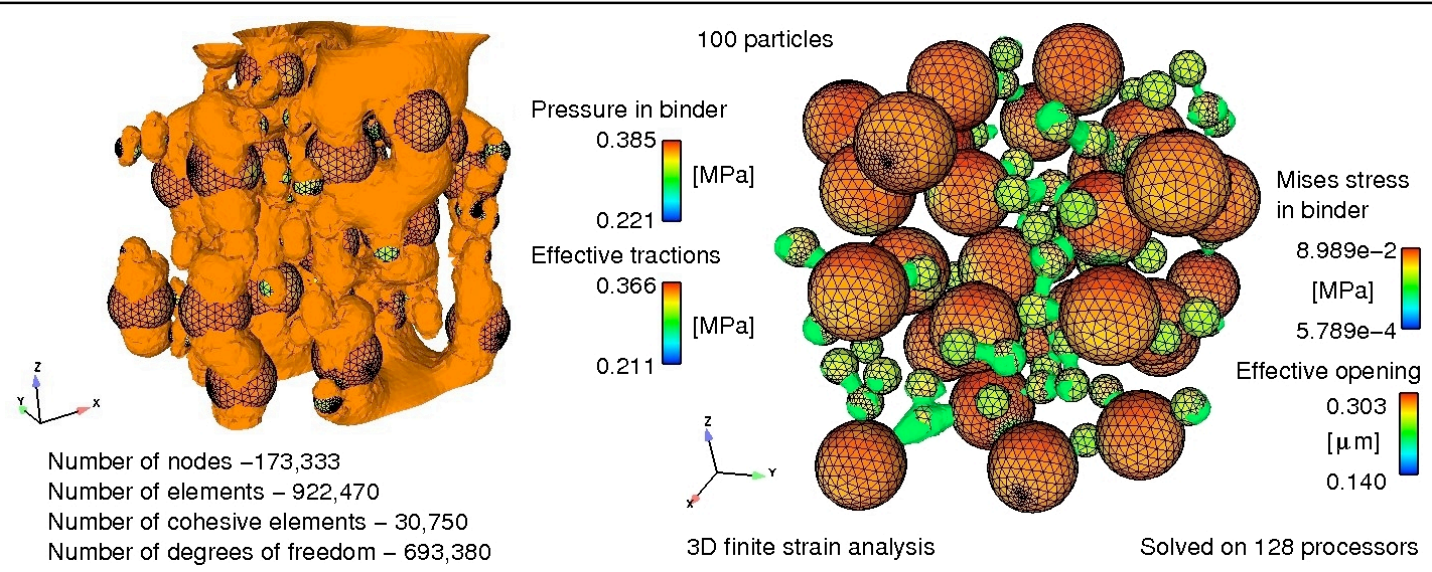

Fig. 3.5.1: Multiscale analysis of constitutive response of reinforced elastomer. Figure on left shows effective tractions along particle matrix interface together with pressure isocontours in binder representing load transfer through unit cell. Figure on right shows effective opening displacement with Mises stress concentrations in binder, where matrix tearing would likely occur.

\section{Integrated Simulation Codes for Structural Response}

Substantial progress has been made in the further development and integration of the explicit (Rocfrac) and implicit (Rocsolid) structural solvers, which are part of the integrated rocket code. 


\section{Rocsolid (Namazifard)}

One of the required components in simulating solid rocket motors is computing the structural response of the propellant, case, liner and nozzle. Our parallel structural analysis code Rocsolid, employs a finite element discretization of the problem domain using unstructured meshes. Dynamic problems are solved using the implicit Newmark time integrator. The linear matrix equations encountered within the Newton iterations at each time step are solved using a scalable parallel multigrid solver. The case of the rocket is modeled by using enhanced assumed strain solid elements. The regression of the propellant is implemented by using an arbitrary Lagrangian-Eulerian (ALE) approach in which the mesh moves to allow for the dynamically changing geometry. The code is written in Fortran90, and uses MPI to perform interprocessor communications.

Large deformations are formulated using strainsstresses and their rates defined on an unrotated frame of reference. This model predicts physically acceptable re-

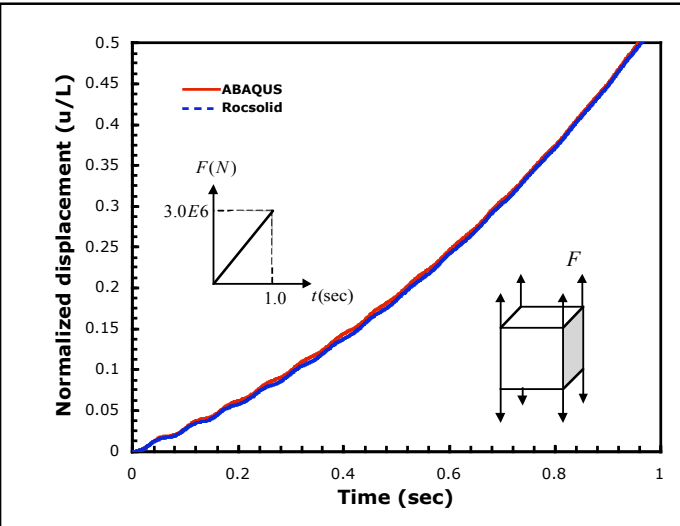

Fig. 3.5.2: Comparison to Abaqus simulation for nonlinear composite material model sponses for homogeneous deformations of exceedingly large magnitude. The implemented numerical algorithm is suitable for the large strain increments, which may arise in the implicit solution of the global equilibrium equations, employed in Rocsolid. The finite rotation effects on strain-stress rates are separated from integration of the rates to update the material response over a time step. This formulation is also adopted in large-scale finite element codes, including NIKE, Abaqus-Standard, and Abaqus-Explicit.

Rocsolid features nonlinear composite constitutive models to predict damage evolution in solid propellant. Three different constitutive models for solid propellant materials are available in Rocsolid. The first model describes a porous viscoelastic material, i.e., the effect of damage-induced porosity on the constitutive behavior of a solid propellant. The presence of porosity is considered to be a result of complete particle dewetting. The material model is developed by considering a unit cell containing a spherical void under macroscopic axisymmetric stressing. In view of the complexity of the calculations, the pure matrix material is assumed to be described by the standard isotropic linear viscoelastic model. The model is based on small strain formulation. Using the correspondence principle of linear viscoelasticity, a constitutive potential is devised for the response of the porous medium in the transformed domain. By inverting the associated constitutive equation from the Laplace transform domain, the timedependent response of the porous medium under timedependent loads is established.

The latest version of Rocsolid includes a mechanismbased constitutive model using state-of-the-art homogeniza-

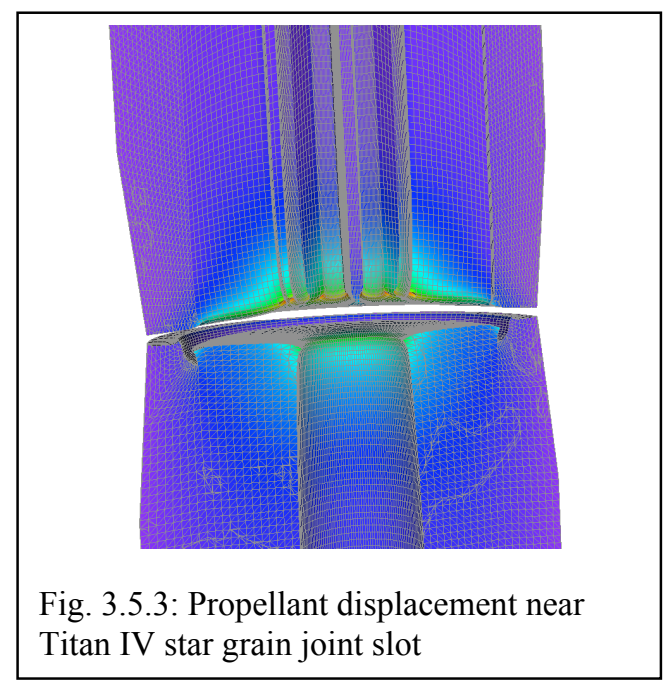
tion procedures for nonlinear composites and strain hardening formulation. The model accounts for the evolution of the microstructure upon straining as is demonstrated by void formation and growth. Since the model addresses the underlying material deformation mechanisms, the model parameters can be determined experimentally. Using this model, the prediction that there are three regimes of deformation for a typical solid propellant material, namely linear, hardening, and softening agrees well with experiment. 
Also in qualitative agreement with the experiment is the dependence of the response on the strain rate. This model is an improvement for an earlier version, which was based on time hardening formulation.

\section{Verification of the Material Models Implementation}

The solid propellant material models were originally implemented and tested in Abaqus through "User Material" subroutines. In order to verify the numerical procedures in Rocsolid and test the implementation of these constitutive models, results are compared for a uniaxial tension problem. Figure 3.5.2 shows the displacements computed by Rocsolid and Abaqus using the nonlinear composite material model (with strain hardening formulation). The results are in complete agreement through out the solution of uniaxial tension test problem.

\section{Application to Titan IV Slumping Problem}

The damage constitutive models available in Rocsolid were used to simulate Titan IV SRMU PQM-1 slumping problem. The generated mesh for this rocket motor consisted of 1.8 million elements. A coupled fluid-structure-combustion simulation was performed, where the solid propellant was modeled using a nonlinear composite material model and the predictor-corrector iterations were employed to enhance the stability of the coupling algorithm. Figure 3.5.3 shows the computed displacement near the star grain joint slot.

\section{Other Enhancements to Rocsolid}

Other new enhancements to Rocsolid include development of a 10-node tetrahedral element su pporting large deformations analysis with all the current material models. Patran can now be used for tetrahedral mesh generation together with Rocsolid Preptool to prepare necessary input files. Mesh partitioning is done by Metis for multi-processor computations.

P. Nakshatrala, K. Nakshatrala, Hjelmstad and Namazifard continue to work on the fluid-solid interaction problem. Our constraint that the fluid code can only utilize Direchlet boundary conditions have hampered our efforts. Nonetheless, we believe that we have developed a promising algorithm that is suitable for the problem at hand. It relies on the minimization of the momentum flux difference across the fluid-solid interface. We have implemented the algorithm for the heat conduction problem in a fully uncoupled staggered approach and are currently studying its consistency and convergence characteristics. Once this study is completed we will test the algorithm in a 1-D fluid-solid simulation.

Tonks, Besudoin and Namazifard are investigating the effects of randomness in polycrystal plasticity simulations. By adding a small amount of randomness to the uniform velocity gradi-

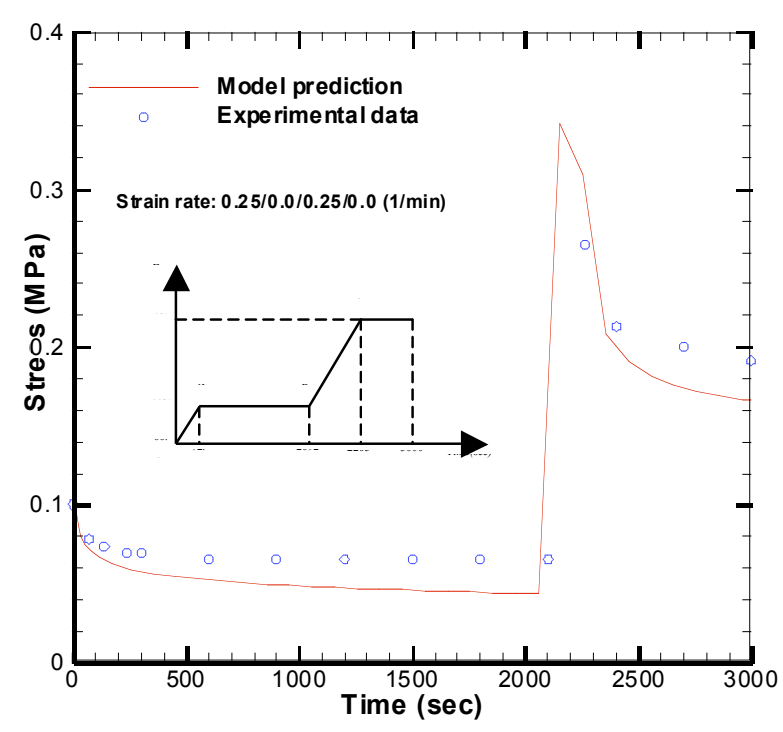

Fig. 3.5.4. Model prediction under complex loading course compared to experimental data for high elongation solid propellant material. From $O$ to $A$, specimen is strained 0.03 at constant strain rate $0.25 / \mathrm{min}$. From $A$ to $B$, strain is held constant and stress relaxes. From $B$ to $C$, specimen is strained from strain 0.03 to strain 0.5 at constant strain rate $0.25 / \mathrm{min}$. From $C$ to $D$, strain is held constant and stress relaxes.

ent that is used in the Taylor model, we are able to produce results that are observed in laboratory experi- 
ments, e.g. we do not obtain tumbling solutions which imply the crystals endlessly rotate, rather the crystal rotatons in our simulations are dampened, eventually defining a steady-state texture. Such findings are consistent with costly multiscale finite element computations. Currently, we are investigating the effects of the randomess on the response and the use of the Kuramoto model to better explain our response.

\section{Rocsolid Implementation of Constitu- tive Modeling and Fracture (Xu, Aravas, Sofronis)}

A nonlinear, "finite-strain," 3-D constitutive model for solid propellant materials whose microstructure continuously evolves under load has been developed and implemented in Rocsolid through an Abaqus type "User Material" subroutine. The model is based on rigorous homogenization theory for nonlinear viscous-matrix composites, accounts for particle dewetting and void growth, involves a "back-stress" concept for properly treating the viscous relaxation, and describes the viscoplastic history of the propellant deformation without resorting to explicit time dependence. The model predictions for simple uniaxial tension over a wide range of applied strains and strain rates and for a complex loading history that involves relaxation at constant load (Figure 3.5.4) are in good agreement with the experiment even for high elongation solid propellants. The model has been used to simulate propellant ignition triggered by void collapse under dynamic insult. Figure 3.5.5 shows contour plots of the temperature distribution around a collapsing void in a cylindrical bar impacted at $50 \mathrm{~m} / \mathrm{s}$. The distribution has been obtained by solving the coupled thermomechanical problem in which the viscous dissipation is used as a heat source. The temperature just above the collapsing void increases from $273 \mathrm{~K}$ to $1000 \mathrm{~K}$ in $0.001 \mathrm{~s}$ after the impact. The model is currently used to investigate the small scale yielding behavior ("boundary layer formulation") of a solid propellant at a crack tip. Possible scaling of the solution is currently being explored in an effort to establish a fracture criterion based on crack propagation resulting from severe damage accumulation at a notch root.

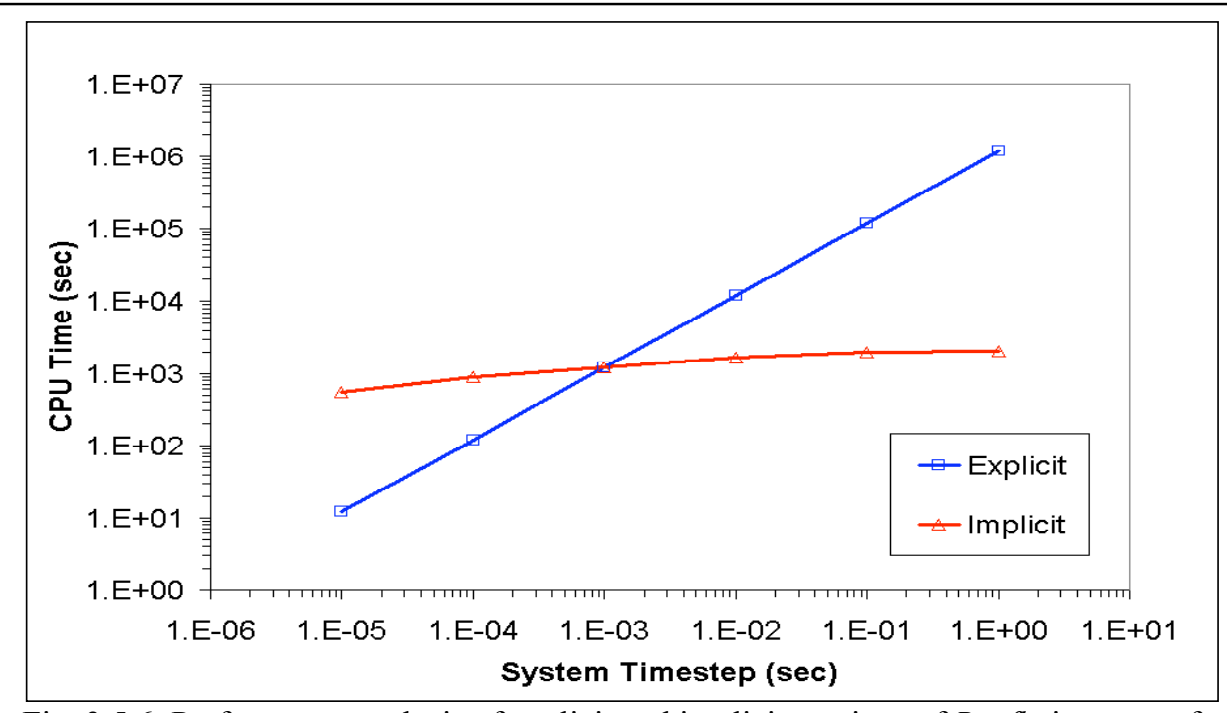

Fig. 3.5.6: Performance analysis of explicit and implicit versions of Rocflu in terms of system time step size (at which information is passed across the fluid/structure interface). For small time step sizes, explicit approach is more efficient, but as system time step increases (flutter problem), cross-over takes place and implicit implementation of fluid solver becomes method of choice. 
3-D Flutter using Rocstar (Dewey, Haselbacher, and Breitenfeld)

In this collaborative project with the CSAR Fluids Group, we investigate the ability of Rocstar, and in particular, of an implicit version of the finite element structural solver Rocfrac and the unstructured finite volume solver Rocflu to simulate the flutter response of wings. Special emphasis has been paced on the simulation of the AGARD Wing flutter problem (Yates et al., 1963), considered as the reference problem for 3-D aeroelastic codes. A substantial amount of effort was placed in the development of the implicit fluid solver, since it quickly became apparent that the slow time scales involved in flutter problem (associated with the first few vibration modes of the wing) render the explicit treatment of both solid and

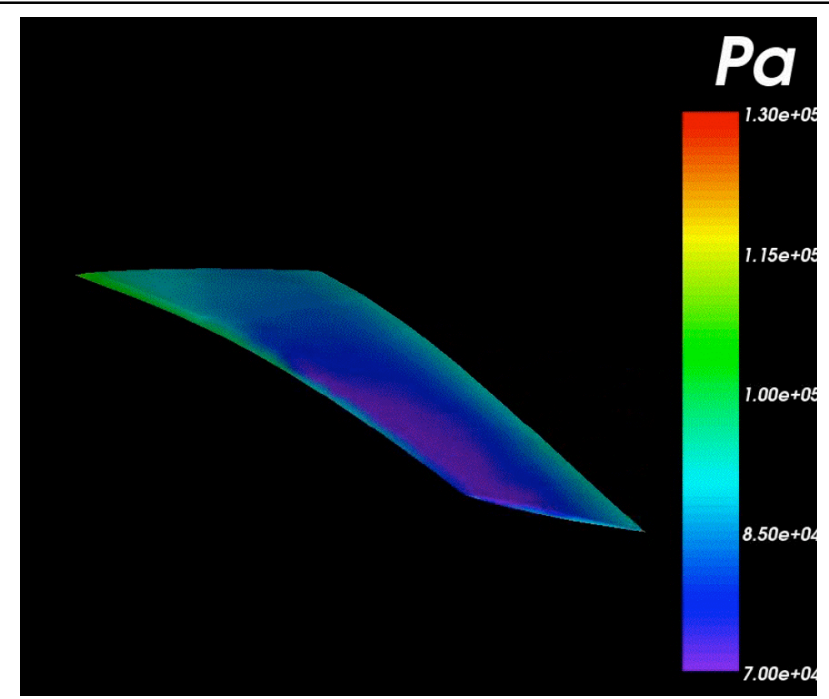

Fig. 3.5.7: Surface pressure and deformed shape of AGARD wing in flutter regime. fluid solver impractical (Figure 3.5.6).

An example of the aeroelastic flutter analysis is shown in Figure 3.5.7, showing the large bending and torsion deformations (captured with the implicit structure solver using mixed-enhanced finite elements) experienced by the wing in the flutter regime.

\section{Stable Implicit/explicit Coupling Schemes for Fluid/structure Interaction (Jaiman, Geubelle, Jiao and Loth)}

A new loosely-coupled staggered procedure for modeling unsteady fluid-structure interaction has been developed, which relies on a higher-order Combined Interface Boundary Conditions (CIBC) treatment on the interface response for improved accuracy and stability of fluid-structure coupling. Traditionally, continuity of velocity and momentum flux along interfaces is satisfied through algebraic jump conditions in a staggered fashion. More specifically, velocity continuity (Dirichlet) conditions are imposed on the fluid side and momentum flux (Neumann) conditions are imposed on the solid side in the staggered procedure. In this type of treatment, the interface undermines stability and accuracy of coupled fluidstructure simulations. By utilizing the CIBC technique on the Dirichlet and Neumann boundary conditions, the staggered procedure can be constructed with the same order of accuracy and stability of standalone computations. Introduced correction terms for velocity and momentum flux transfer can be
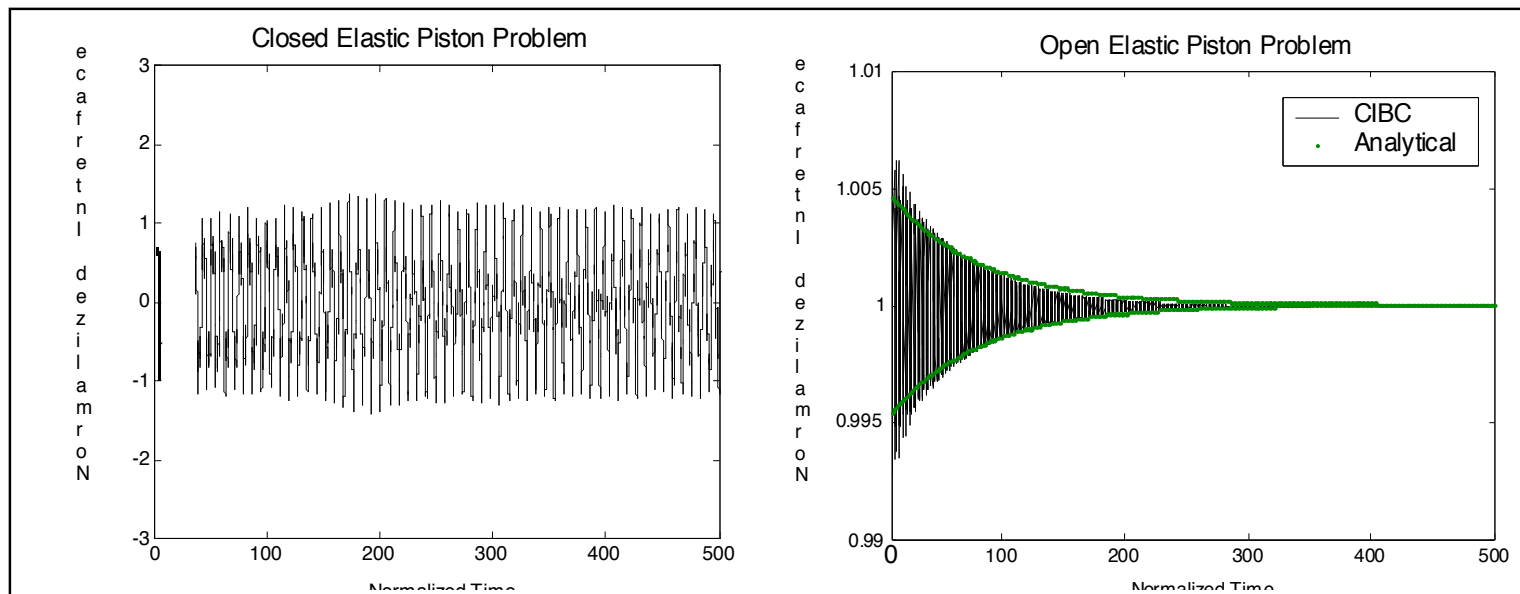

Fig. 3.5.8. Time evolution of interface pressure for closed (left) and open (right) elastic piston problem, showing excellent stability and precision properties of CIBC scheme. 
explicitly added to the standard staggered time-stepping stencil so that the discretization is well-defined across the interface. A range of coupling parameter that yields stable and accurate CIBC discretization is found by 1-D numerical experiment of closed- and open-elastic piston model problems (Figure 3.5.8). Finally, we demonstrate the validity of the 1-D analysis for a 2-D application involving a subsonic flow and thin-shell structure.

\section{Asynchronous Multidomain Variational Integrators for Nonlinear Problems (Gates, Matous and Heath)}

This work is focused on integration and coupling algorithms based on variational techniques for solving linear and nonlinear mechanics problems, with domain decomposition for parallel computing. Variational integrators preserve many properties of the continuous system, and have a bounded error on energy, and are thus superior methods for long-term integration (Figure 3.5.9). They also naturally accommodate constraints using Lagrange multipliers, which we use for the domain decomposition. We have developed asynchronous symplectic integrators, where each domain has its own time step, using a smaller time step where required by stability and accuracy constraints, and a larger time step where allowed. The various integrators are analyzed using linear and nonlinear springs.

Stability and Prevision of Coupled Transient Thermal Analysis (Roe, Haselbacher, Geubelle and Jaiman)

In this project, we investigate the effect of the treatment of the interface in the numerical analysis of fluid/structure thermal problems. The analysis is performed using the analytical Godunov-Ryabenkii's stability method and a 1-D loosely coupled thermal solver based on the finite difference, finite volume or finite element treatment. Recent emphasis has been placed on adapting the CIBC coupling procedure described in this Section in Jaiman et al. to the coupled thermal problem. It was shown that substantial improvements could be achieved both in terms of the stability and precision of the coupled thermal scheme.

\section{Materials Research}

Multiphysics Solver for Burning of Damaged Solid Propellant (Srinivasan, Matous, Geubelle and Jackson)

Previous research efforts at CSAR (Rocfire) have focused on the development of an unsteady 3-D solver to simulate the burning response of heterogeneous propellants. However, these efforts excluded the effect of solid propellant deformations on its burn characteristics. The current study focuses on the behavior of burning solid propellants under large thermo-mechanical strains, observed in the vicinity of the burning propellant surface. In lieu of the complex topologies encountered, a level-set approach is adopted to describe the regressing surface. A par-

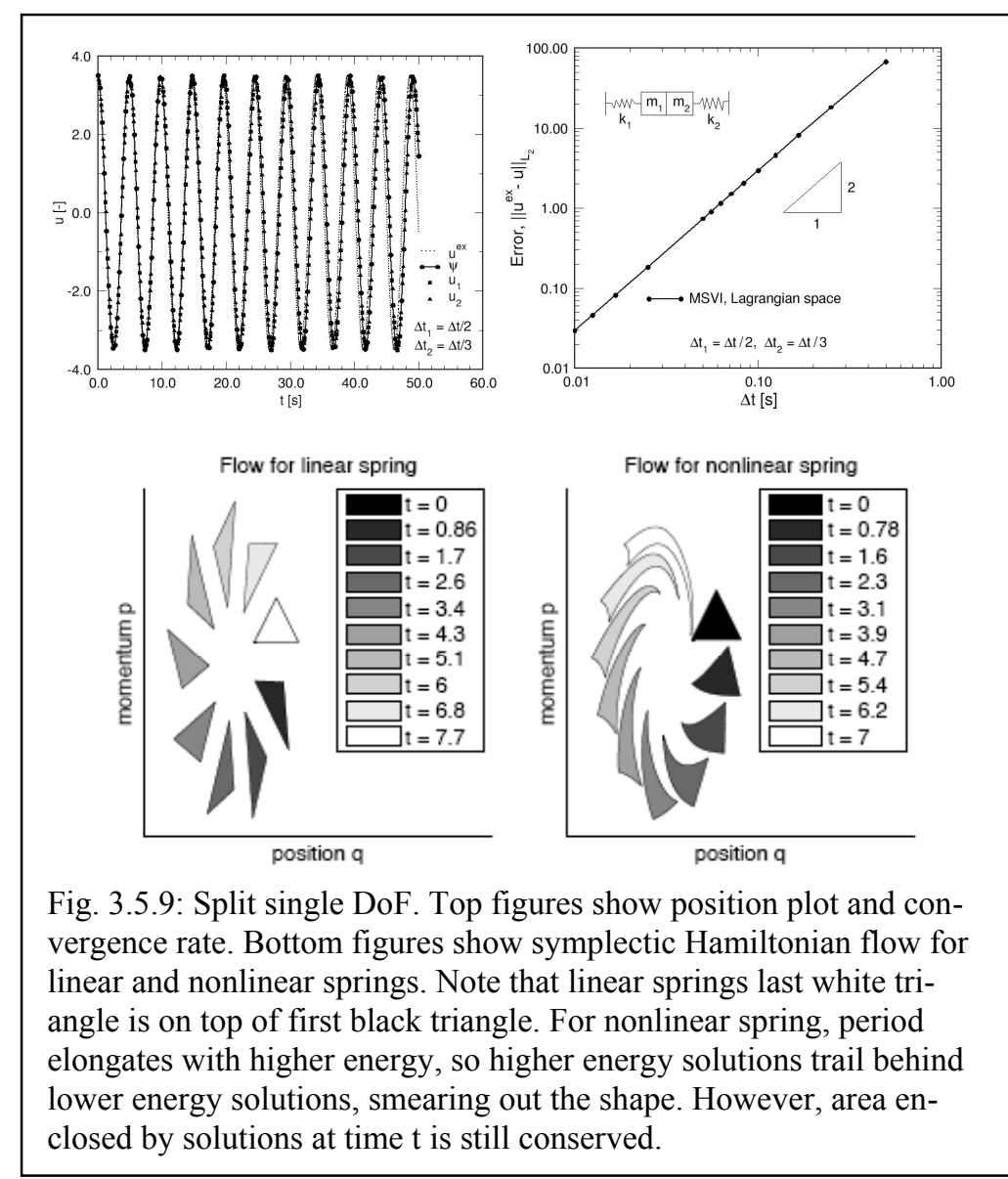


tition of unity (GFEM) approach coupled with the level-set approach is used to model the structural response of the AP/Binder interface. The highlight of this scheme is the evolution of the regressing surface without remeshing. The large thermomechanical strains in the incompressible binder demand a sophisticated three-field $\mathrm{Hu}-\mathrm{Washizu}$ formulation for accurate results. The three-field formulation has been extended to the GFEM with very small changes in convergence rates. The structural solver is coupled (staggered) to a transient thermal solver and was successfully applied to a random 2-D pack (Figure 3.5.11). Future works include interaction of the regressing surface with a dewetted AP/Binder interface.

\section{Homogenization Theory for Multiscale Modeling of Damage in Solid Propellants (Matous, Kumar, Collins and Geubelle)}

Substantial progress has been made in the area of the multiscale numerical modeling of the damage evolution in solid propellants (Figure 3.5.1 above). The framework of hierarchical numerical modeling has been formulated and the 3-D numerical apparatus, including the implicit cohesive model, has been created for nearly incompressible solids subjected to finite deformations. This model allows in detail studies of damage evolution in solid propellants. Furthermore, we have developed the highperformance parallel multiscale solver, which is used to investigate more complex and representative

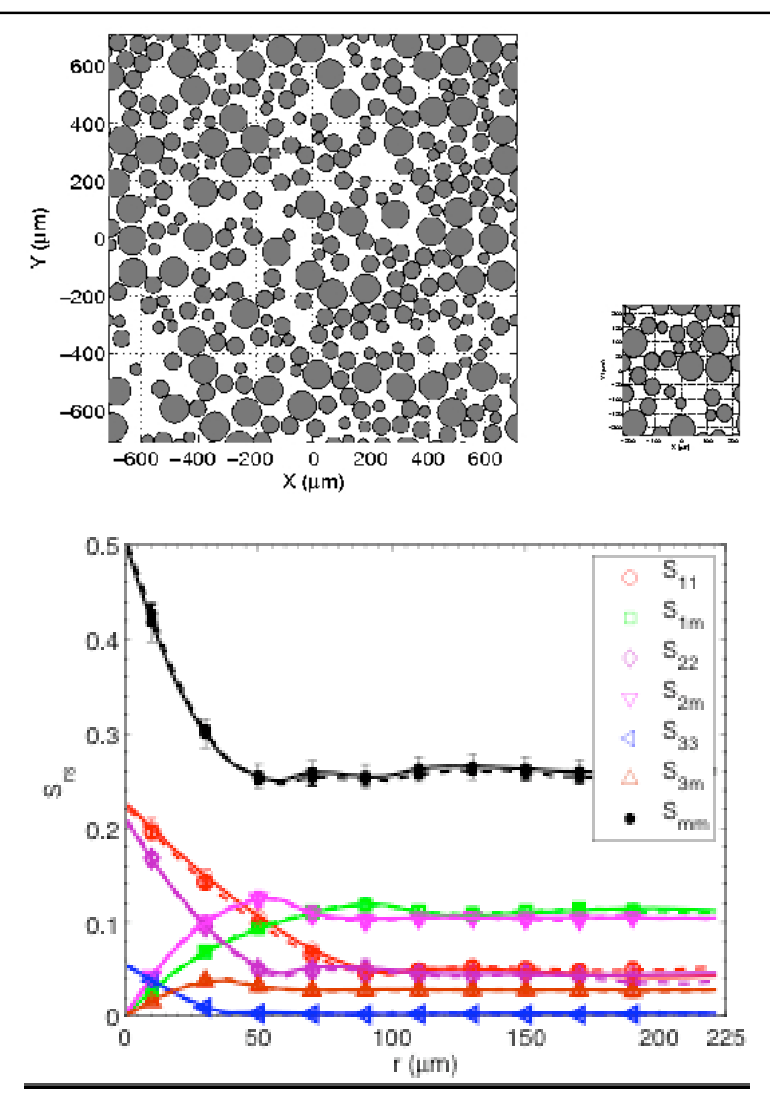

Fig. 3.5.10: Top figure shows pack and cell geometries with $50 \%$ volume fraction. Bottom shows comparison of second order probability functions between pack and cell, respectively. Note large reduction of computational space.

3-D unit cells (Figure 3.5.10). The mathematical theory of homogenization has been employed and implemented into 3-D code. This theory with natural scale bridging mechanism is used in predicting both meso and macroscale behavior of the solid propellant under progressive debonding. Moreover, we have created the module to compute the probability functions of first and second order to characterize the pro-

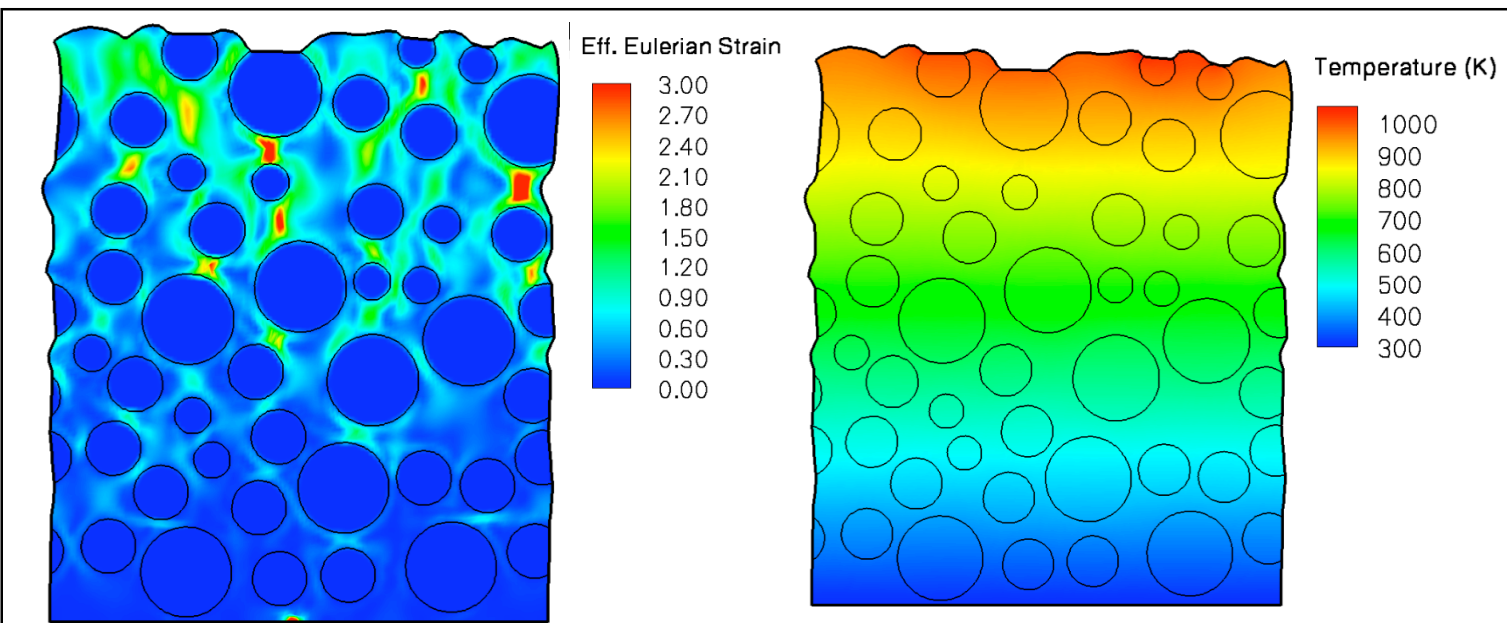

Fig. 3.5.11. Thermal and pressure-driven deformation in a burning heterogeneous solid propellant (left) and corresponding temperature distribution (right). 
pellant. Such characterization is used in the unit cell reconstruction procedure. Thus, computational cells with the same and/or similar statistics as the original pack generated by Rocpack are now constructed.

Combustion of Deformable Heterogeneous Solid Propellants (Sninivasan, Matous, Jackson and Geubelle)

This work builds on

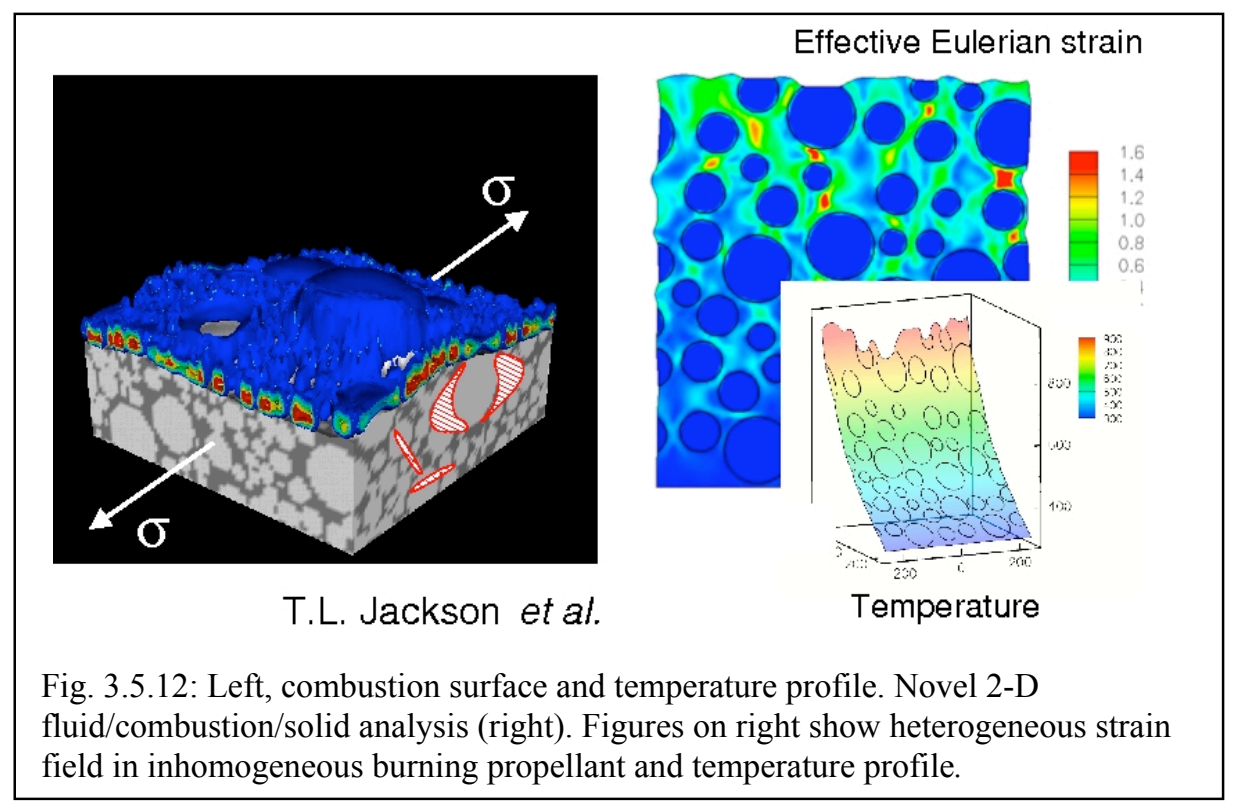
the capabilities of our combustion group, where the large-scale computations of propellant combustion are carried out, but on solids without deformation. Here we want to account for deformation due to the external thermo-mechanical loads and connect our damage evolution modeling with combustion phenomena (Figure 3.5.12). Thus, we have derived 2-D finite strains generalized finite element thermomechanical solver for modeling of combustion processes in the deformable heterogeneous propellants. The framework is based on the partition of unity method, which allow us to model the regressing combustion front without need of complicated remeshing. The partition of unity method relies on introduction of special local enhancement functions, which are used to enrich the finite element approximation space. The combustion interface is then advanced by the level-set method. The partition of unity method is also employed in modeling the particle/matrix interfaces. The finite element approximation is constructed using the $\mathrm{Hu}$-Washizu variational principle in order to eliminate the spurious pressure oscillations due to the nearly incompressible behavior of a blend.

\section{Multiscale Cohesive/interfacial Modeling (Kulkarni, Matous and Geubelle)}

Over the past year, we have derived and implemented a novel multi-scale cohesive modeling framework, incorporating the important physical processes in the adhesive/interfacial layer and allowing for the natural scale bridging (Figure 3.5.13). Such theory is advantageous in cohesive failure mechanisms and can capture physical phenomena missed by the most conventional approaches. The proposed method for coupling the macro- and micro- domains relies on a hierarchical decomposition of the approximation space. At the macroscale, we define the cohesive layer kinematics in order to obtain the strain of the adhesive layer in terms

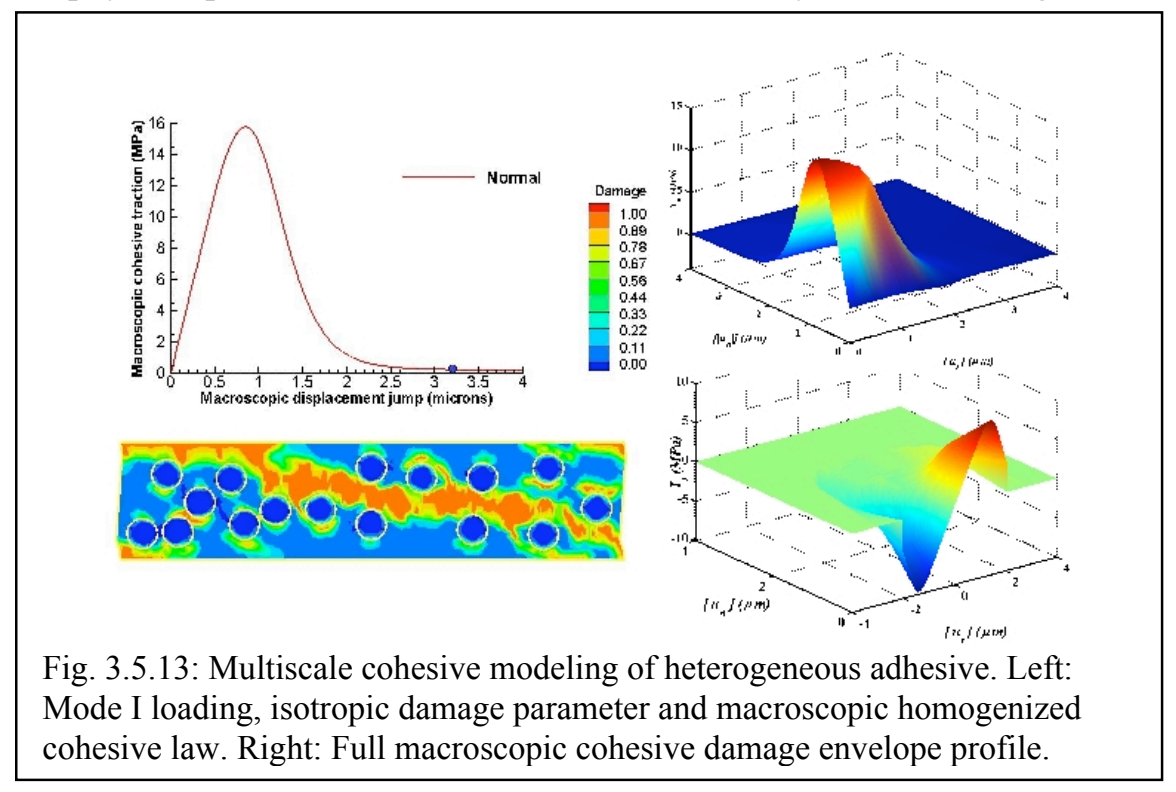


of the displacement jump, across the cohesive faces. The material periodicity is assumed in the discontinuity plane and an asymptotic expansion is employed, whereas the intrinsic cohesive length scale, defined by the layer thickness, is introduced and the space dimension reduction is performed perpendicular to the interface. The macroscopic displacement jump across the adhesive layer is then used as a forcing term in order to derive the homogenized cohesive traction-separation law used at the macro-scale. This framework is very advantageous in modeling the heterogeneous insulation layer, for example, and allows for accurate mode mixity.

\section{Multiscale Modeling of Damage in Solid Propellant using RVE (Inglis, Patel, Kramer, Geubelle,} Matous, Tan and Huang)

Multiscale modeling of a closely packed particulate composite such as solid propellant requires the determination of a Representative Volume Element (RVE) which is taken as the microscale base cell on which computations are performed. Accuracy of the computational results relies on the choice of an RVE that is representative of the bulk material in geometrical arrangement (e.g. volume fraction, particle distribution) and in mechanical and failure properties (e.g. modulus, evolution of damage). The material response of packs containing a 50\% volume fraction of 100 micron particles is shown in Figure 3.5.14. Three pack sizes of dimensions four, eight and

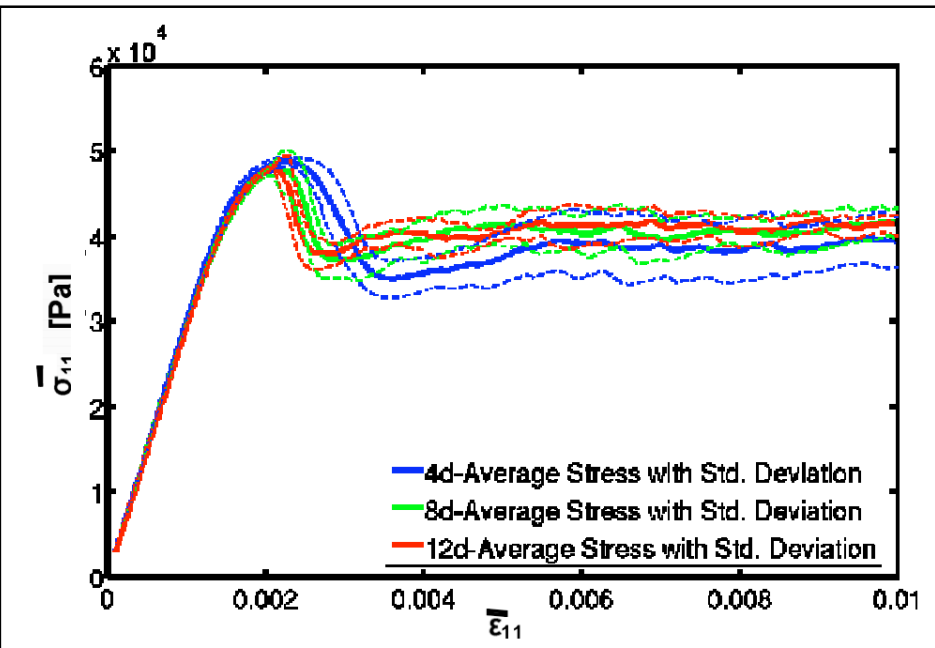

Fig. 3.5.14: Macroscopic stress-strain response for different size packs under uniaxial strain.

twelve times the particle diameter $(4 \mathrm{~d}, 8 \mathrm{~d}, 12)$ were considered, with ten randomly generated packs of each size. The curves show characteristic peak and plateau stresses, with the $8 \mathrm{~d}$ and $12 \mathrm{~d}$ results substantially similar. Damage proceeds through the formation of a series of localization bands across the domain, shown in Figure 3.5.14 for three different 8d packs. While the specific location of bands differs from pack to pack, the type and extent of damage is consistent across all the packs.

\section{Multiscale FE Code for Dynamic Properties of Heterogeneous Materials (Breitenfeld and Geubelle)}

The area of dynamic multiple-scale modeling, in which the effects of inertia influence both the fine and coarse scales, is being investigated. The model at the fine or microscale encompasses the nature of the inherent inhomogeneity of propellants and energetic materials by modeling the matrix and particles
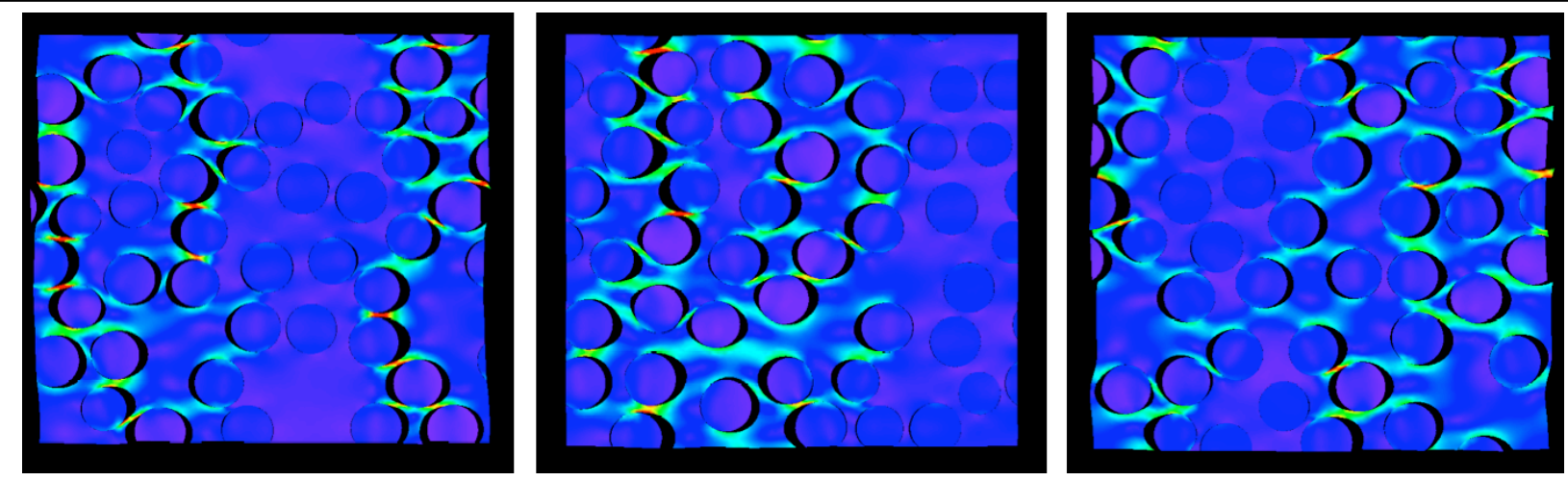

Fig. 3.5.15: Three examples of deformed $8 \mathrm{~d}$ packs, showing formation of localization bands. 
using a periodic representative volume element. The constitutive material law for the micro-scale components is typically nonlinear, and damage between the interface of a particle and the matrix is captured by the use of a traction-separation law incorporated within the cohesive elements. Using this model, the effect of inertia on particle dewetting is studied at the microscale. Figure 3.5.16 shows the effects of strain rate on a representative volumetric element containing 100 particles (see inset) under tension; notably, at the higher strain rate the onset of particle de-wetting is delayed in comparison with slower strain rates. Work is being completed in coupling the separate time scales of the coarse and fine scales in order to fully couple

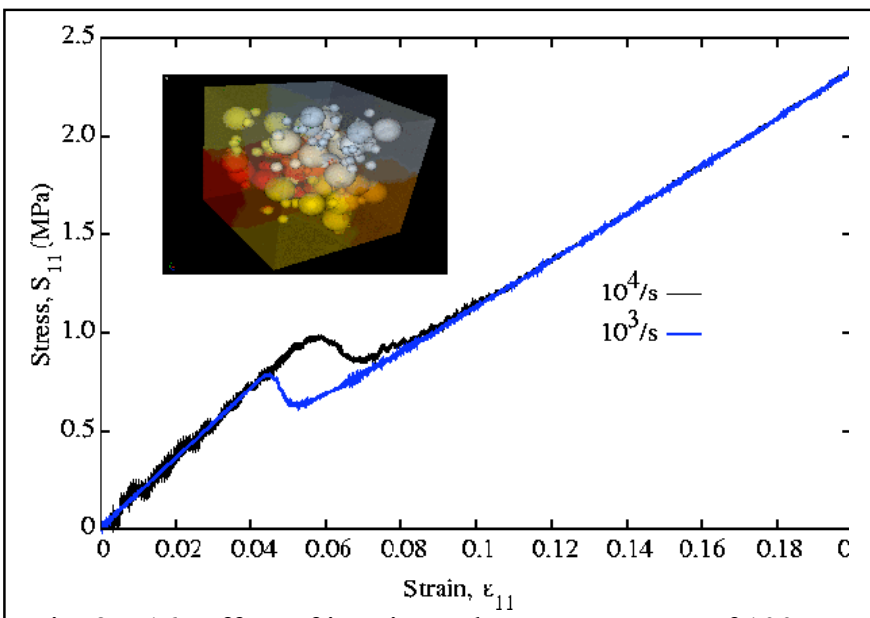

Fig. 3.5.16: Effect of inertia on damage response of 100particle RVE subjected to dynamic uniaxial macroscopic strain. the multiple-scale modeling.

Constitutive Model of Particle Dewetting for Energetic Materials (Huang, Dong, Tan, Liu, and Lu)

Our group has been collaborating with Los Alamos National Laboratory, Sandia National Laboratories (both Albuquerque and Livermore) and Lawrence Livermore National Laboratory to develop the constitutive model for energetic materials accounting for the effect of particle dewetting from the binder matrix.

Debonding of particle/matrix interfaces can significantly affect the macroscopic behavior of composite material. We have used a nonlinear cohesive law for particle/matrix interfaces to study interface debonding and its effect on particulate composite materials subject to uniaxial tension. The dilute solution shows that, at a fixed particle volume fraction, small particles lead to hardening behavior of the composite while large particles yield softening behavior. Interface debonding of large particles is unstable since the interface opening (and sliding) displacement(s) may have a sudden jump as the applied strain increases, which is called the catastrophic debonding (Figures 3.5.17 and

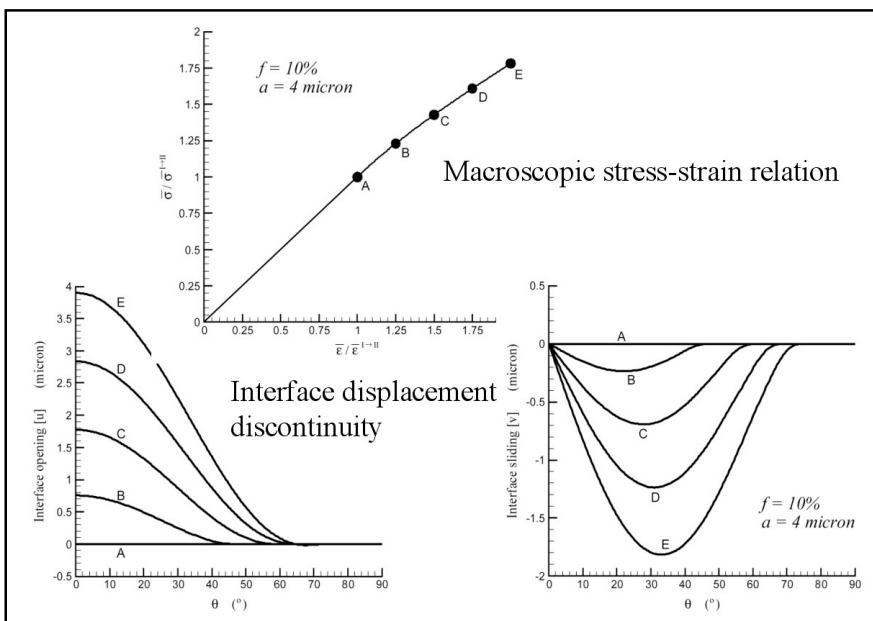

Fig. 3.5.17: Hardening behavior is observed in composite materials containing small particles 3.5.18). A simple estimate is given for the critical particle radius that separates the hardening and softening behavior of the composite.

\section{Rate Dependent Properties of Metals (Beaudoin and Padilla)}

The modeling effort for metals has focused on the study of rate-dependent and anisotropic properties. Models are derived with a physical basis and address the strain rate and temperature dependence of metal plasticity, effect of microstructural length scale, and impact of grain boundaries on deformation. The detailed mesoscale models have been condensed into relations appropriate for "engineering scale" analysis of components. The temperature and strain rate dependent properties of the barrel-rolled D6AC steel case 
material have been characterized, and the hardening response of steels - as well as other metals -- at large strains has been derived through consideration of an intrinsic length scale. In 2003, this CSAR modeling program has been augmented to include an experimental component through an award under the Stewardship Science Academic Alliances Program of the DOE (Prof. I. Robinson, PI). This award complements the simulation component of the mesoscale modeling program with fundamental study on the effect of grain boundaries in metal deformation. Through this program, the mesoscale model developed under the ASCI funding has been applied to draw interpretation from experimental studies - all in collaboration with DOE research scientists.

Metals considered in these combined experimental/computation studies are fine-grained polycrystalline $\mathrm{Ag}$ (with Korzekwa, LANL), polycrystalline $\mathrm{Zr}$ (with Kaschner \& Tomé, LANL) and Cu-Nb (with Misra \& Tomé, LANL). The parallel finite element code for mesoscale fracture, also developed under the CSAR program, is presently utilized in a NASA funded program to study a delamination fracture mode in the Al-Li alloy C458. This alloy is under consideration for structural application in the next generation launch vehicle (NGLV). 